determinations have become a routine they have been taken over by other groups.

The Institute is well equipped with a variety of modern instruments and apparatus for biochemical and biophysical work. In addition, the radiobiological investigations require two special kinds of facilities, namely, radiation sources and experimental animals of very high quality. The Radiobiological Institute has at its disposal, besides small $\alpha$ - and $\beta$-sources, a 200-c. cæsium-137 $\gamma$-source for prolonged radiation of experimental animals and a 250-kV 'Maxitron' X-ray unit with circular beam used for short-term radiation. The special tube with which this apparatus is equipped makes it possible to irradiate a number of large animals simultaneously.

Lastly, a neutron generator is in use for measurements in the field of neutron dosimetry, and with this machine small biological objects such as cultured cells or small numbers of mice can be irradiated with neutrons. Cooperation has been established with the Reactor Centre of the Netherlands at Petten, which will make it possible to expose larger experimental animals to fission neutrons.

The laboratory has four specially designed rooms for biological work with higher levels of radioactive isotopes with installations for the removal of airborne and fluid waste.

In the Institute an attempt is made to maintain unusually high standards of health in the experimental animals used for radiobiological research. This policy, in which a great deal of effort and resources is involved, is followed for two reasons. In the first place, in many experiments the animals are exposed to total-body irradiation which greatly reduces their resistance to infection. When pathogenic micro-organisms are present even without causing manifest disease symptoms, there is a risk that the animals will develop the disease after irradiation. The consequent mortality will often prevent the successful conclusion of experiments. In the second place, a very prolonged observation period is required for the investigation of the so-called late effects of radiation and of isotope intoxication. These long-term experiments usually extend over the entire life-time of the animal. In the past, the health of the ordinary laboratory animals (conventional animals) often was so poor that most of them died of specific diseases at 'middle-age' or earlier, even without being exposed to other injurious factors.

The opinion held at the Radiobiological Institute is that long-term experiments with insufficiently selected animals will result only in loss of time and money. A start was therefore made some years ago, in co-operation with the Medical Biological Laboratory RVO-TNO, on a programme of far-reaching sanitation of the animal colonies. The procedure was based on Cæsarean-delivered, hand-fed young animals raised under strict isolation. The hand-feeding has now been abandoned since gnotobiotic animals are available for foster-nursing. The experience obtained so far has led to a modified approach -which is at present being pursued-namely, a multiplestep system for the production of satisfactory animals. These steps range from heavily protected gnotobiotic animals, via specific pathogen-free stocks to lightly protected production units. In cases of infection or other disorders in the production colonies, supplementation and, if necessary, total replacement can be made with animals from the next higher step. This project receives financial support from Euratom, and one of its objectives is to determine what grade of sanitation is optimal for the various kinds of radiobiological work.

The staff of the Institute includes, in addition to two physicists and two biochemists, seven medical graduates with training in different fields.

\section{M. van Putten}

\title{
THE CARNEGIE INSTITUTION OF WASHINGTON
}

\begin{abstract}
$Y^{\text {s }}$ EAR BOOK No. 61 * of the Carnegie Institution of Washington, covering the year ended June 30, 1962, and including the reports of the President, the Mount Wilson and Palomar Observatories, the Geophysical Laboratory, the Committee on Image Tubes for Microscopes, and the Departments of Terrestrial Magnetism, Plant Biology, Embryology, and of Genetics, marks the sixtieth anniversary of the Institution. The Institution's budget is now more than $\mathbf{2 . 8}$ million dollars, all of which is spent on six operating departments, whereas in 1905 the budget was less than 600,000 dollars, of which 302,000 dollars went to ten departments, about half of this being for the Solar Observatory on Mount Wilson, then under construction. Some 130,000 dollars were distributed in grants and nearly 30,000 dollars on subsidizing publications.

Experiments on maize had already begun, as had cytological research on Drosophila, and the Department of Genetics is still finding these lines of investigation productive. During the year, Dr. Barbara MeClintock confirmed the hypothesis that there is a high probability that genic control systems in maize and bacteria act in similar fashion. In the meantime, the group working on the application of cytology to genetics found that direct chromosomal breakage occurs in Tradescantia root tips in presence of 5-bromodeoxyuridine, which acts by modifying the base sequences in nucleic acid rather than the phosphate-sugar helixes attacked by deoxyribonuclease, and that Golgi bodies, one of the types of cytoplasmic organ-
\end{abstract}

* Carnegie Institution of Washington. Year Book, No. 61. July 1, 1961June 30,1962 . Pp. $x i+112+526+21$ plates. (Washington, D.C.: Carnegie Institution of Washington, 1962.) elle, exhibit different forms in the progression of microsporogenesis in Tradescantia. The Department has also established the molecular weight of the deoxyribonucleic acid of a bacteriophage known as $T 5$ by first establishing an ingenious pair of scales by analysing the deoxyribonucleic acid fragments of another phage (T2), and has found that the molecular characteristics of the deoxyribonucleic acid of phage lambda differ remarkably from those of other deoxyribonucleic acids, possessing a broad range of denaturation temperatures. The Department is now being terminated as a separate administrative unit and staff remaining in the Genetics Research Unit will cooperate with the new Laboratory of Quantitative Biology which is being organized.

In the Department of Plant Biology attention is still directed on the functions of chlorophyll and the accessory pigments in photosynthesis in which it has been interested since 1911. The persistence of the chemically unidentified material previously formed by illumination of chlorophyll $a$ has been measured, and by measuring oxygen exchange of Swiss chard chloroplasts four separate effects of light, each with its characteristic action spectrum, have been distinguished. Using the new technique of electron paramagnetic resonance, two distinctly different light-induced resonances have been observed, and it is concluded that the rapid-decaying signal, seen only when cells are illuminated, is associated with chlorophyll and arises from the 'primary' act of photosynthesis. Any inhibition of oxygen evolution, as by $3-\left(3^{\prime}: 4^{\prime}\right.$-dichlorophenyl)1 : 1-dimethyl urea, or by limiting the manganese-ion concentration in the growing medium, produces an enhanced rapid-decaying signal. Moreover, besides the 
evidence that chlorophyll is the source of one type of free electrons in an intact photosynthetic organism and that plastoquinone is the site of another type, the two types of signal have been correlated with the evolution of photosynthetic oxygen. Some particularly interesting studies of the photosynthetic rates of six races of Mimulus originating in diverse climates and altitudes indicated that climatic races within the same species may show differential patterns of response linked with variations in internal physiology.

The Department of Terrestrial Magnetism, in which the emphasis of the programme has markedly changed from earlier years, apart from studies of the intensity of the charged particles in the Van Allen trapped-radiation belt (as recorded during the transits of satellite Explorer VII through the belt between 1959 and 1960), was concerned with the application of physical techniques to a wide range of geophysical and biological problems. Using a simple and effective new procedure, it has been demonstrated that the deoxyribonucleic acid-like ribonucleic acid comprises about 1 per cent of the total ribonucleic acid of bacterial cells, and that it has a half-life during active synthesis of about $2 \mathrm{~min}$. If this ribonucleic acid is, in fact, the template for protein synthesis, a single mole. cule acts catalytically for the synthesis of many polypeptide chains. The method can also be used to exploit the specificity in the hybridization process, which depends on long regions of complementary nucleotide sequences in molecules of ribonucleic acid and deoxyribonucleic acid and it can also be used in studies of the transeription of genetic information and of differentiation.

With support of the National Science Foundation, the Department is establishing a new Carnegie Radio Astronomy Station in Argentina, and it is also constructing an interferometer array from parabolic disk antennæ to obtain precise positions of radio-noise sources in the sky. During the period covered by the report, the observational programme in radio astronomy continued and observations of the hydrogen gas content at the centre of our Galaxy confirmed that the motions of hydrogen close to the Galactic centre are complex and that the hydrogen gas is expanding as well as rotating. A major co-operative project of the Seismic Studies Group was an intensive study of the Earth's crust in Maine which demonstrated the very real limitations imposed on our ability to learn about crustal 'layers' by the customary methods of explosion seismology, and its findings postulate appreciably less granitic material than is customarily thought to be in a continental crust. The Group concerned with the radioactive dating of rocks participated in an exchange with the Geological and Mineralogical Institute of the University of Kyoto and has compiled a new map of agedistribution in erystalline basement rocks of North America. The Department is also associated with the work of the Committee on Image Tubes for Telescopes which has now reached a stage when a real gain in research work over the best photographic plates is just beginning to be realized by means of convenient (and permanent) scaled-off image tubes.

The Geophysical Laboratory focused much of its work in experimental petrology during the year on pyroxene minerals and the study of phase equilibria at higher pressures. Additional experimental data for constructing concepts of the Earth's mantle and crust are being obtained from an examination of the melting relations of silicates at high pressures. Much effort was expended on studies of the mineralogy of meteorites, and new investigations in organic geochemistry included analysis of Precambrian carbonaceous materials. Fatty acids were isolated from rocks as old as 500 million years, including myristic, palmitic and stearic acid, and the same acids have been isolated from recent sediments but with a marked difference in the relative proportions of acids. Much the longest of the departmental reports, that from the Geophysical Laboratory, includes summaries of more than fifty investigations.

At the Mount Wilson and Palomar Observatories an extensive study of the accumulated magnetograms to classify magnetic regions and correlate them with optical and radio phenomena showed that the unipolar magnetic regions of the Sun correlate in position with the calcium absorption phenomenon observed spectroscopically. Studies of the spectra of the major planets were commenced and two lines of the hydrogen molecule were found in the spectrum of Saturn. The analysis of high-dispersion spectra of Venus afforded evidence of large changes in the temperature of the atmosphere of Venus. Colourmagnitude diagrams have been constructed for many globular and galactic elusters and high-dispersion spectra used to make detailed quantitative chemical analyses of stellar atmospheres. New knowledge was obtained about differences in chemical composition among stars, the correlation of chemical composition and star movement, and fresh evidence on the expansion of the cosmos from studies of nearby galaxies by photoelectric scanning methods. From studies of the orbits around the nucleus of our Galaxy of a large number of dwarf stars, both normal and sub-dwarf types, the time of formation of the Galaxy out of the medium of the universe has been fixed at about 10 billion years ago.

The completion of the new building for the Department of Embryology was a feature of the year in which the Department's most striking progress was in continuation of its studies of the problems of muscle development. Investigation of the cytodifferentiation of embryonic skeletal muscle cells in dispersed cell culture using freshly isolated embryonic muscle cells showed a plating efficiency of about 10 per cent, and in plates cultivated for 10-13 days about 1 in 10 colonies showed unmistakable signs of skeletal muscle cell differentiation. Further tests showed that the medium is altered by the metabolic activity of cells cultured in it, and the results, impressive in themselves, represent something of a technical breakthrough in the task of cell culture. Other investigations include studies of the physiological aspect of frogembryo growth from the stage of the fertilized egg, of the nature of the testicular antigen in induced aspermatogenesis, of the role of deoxyribonuclease II during the metamorphosis of the tadpole, and a comprehensive study of the developing human eye, as well as a study of the vascular pattern of the human uterus with placenta in situ.

\section{VASCULAR AND LYMPHATIC RESPONSES IN INJURY}

SYMPOSIUM on the "Vaseular and Lymphatic
Responses in Injury", organized by the School of
Pathology of the newly established Faculty of Medicine
of the University of New South Wales, attracted pathol.
ogists from all parts of Australia, and was honoured by
the presence of Sir Roy Cameron, of University College
Hospital, London. The symposium was sponsored by Geigy Pharmaceuticals.

The first session on November 2 was devoted to problems of vascular structure and behaviour. The opening paper was given by Dr. A. A. Palmer (Sydney). Dr. Palmer pointed out that blood in capillaries is separated 